\title{
Improvement in verbal learning over the first year of antipsychotic treatment is associated with serum HDL levels in a cohort of first episode psychosis patients
}

\author{
Priyanthi B. Gjerde $\mathrm{e}^{1,2,6}$ (D) Carmen E. Simonsen ${ }^{3,4,7} \cdot$ Trine V. Lagerberg $^{3,7} \cdot$ Nils E. Steen ${ }^{3,4,7} \cdot$ Torill Ueland $^{3,5,7}$. \\ Ole A. Andreassen ${ }^{3,4,7} \cdot$ Vidar M. Steen ${ }^{1,2,6} \cdot$ Ingrid Melle $e^{3,4,7}$
}

Received: 27 October 2018 / Accepted: 16 April 2019 / Published online: 27 April 2019

(c) The Author(s) 2019

\begin{abstract}
To investigate whether changes in serum lipids are associated with cognitive performance in first episode psychosis (FEP) patients during their first year of antipsychotic drug treatment. One hundred and thirty-two antipsychotic-treated FEP patients were included through the TOP study along with 83 age- and gender-matched healthy controls (HC). Information regarding cognitive performance, psychotic symptoms, lifestyle, body mass index, serum lipids [total cholesterol, high-density lipoprotein (HDL) cholesterol, low-density lipoprotein cholesterol, and triglycerides] and antipsychotic treatment was obtained at baseline and after 1 year. The cognitive test battery is comprised of assessments for verbal learning, processing speed, working memory, verbal fluency, and inhibition. Mixed-effects models were used to study the relationship between changes over time in serum lipids and cognitive domains, controlling for potential confounders. There was a significant group by HDL interaction effect for verbal learning $(F=11.12, p=0.001)$, where an increase in HDL levels was associated with improvement in verbal learning in FEP patients but not in HC. Practice effects, lifestyle, and psychotic symptoms did not significantly affect this relationship. Antipsychotic-treated FEP patients who increased in HDL levels during the first year of follow-up exhibited better verbal learning capacity. Further investigations are needed to clarify the underlying mechanisms.
\end{abstract}

Keywords Schizophrenia $\cdot$ Psychosis · Antipsychotics · Lipids · Cognition · Outcome

Vidar M. Steen and Ingrid Melle contributed equally.

Electronic supplementary material The online version of this article (https://doi.org/10.1007/s00406-019-01017-w) contains supplementary material, which is available to authorized users.

Vidar M. Steen

vidar.martin.steen@helse-bergen.no

Priyanthi B. Gjerde

priyanthi.gjerde@uib.no

Carmen E. Simonsen

c.e.simonsen@medisin.uio.no

Trine V. Lagerberg

t.v.lagerberg@medisin.uio.no

Nils E. Steen

n.e.steen@medisin.uio.no

Torill Ueland

torill.ueland@medisin.uio.no

Ole A. Andreassen

o.a.andreassen@medisin.uio.no

Ingrid Melle

ingrid.melle@medisin.uio.no
1 NORMENT, Department of Clinical Science, University of Bergen, 5021 Bergen, Norway

2 Dr. Einar Martens Research Group for Biological Psychiatry, Department of Medical Genetics, Haukeland University Hospital, 5021 Bergen, Norway

3 NORMENT, Oslo University Hospital, 0424 Oslo, Norway

4 Department of Clinical Medicine, University of Oslo, 0424 Oslo, Norway

5 Department of Psychology, University of Oslo, 0317 Oslo, Norway

6 Laboratory Building, Department of Clinical Science, Haukeland University Hospital, P.O. Box 7804, 5021 Bergen, Norway

7 Oslo University Hospital, Ullevaal, Building 49, Nydalen, P.O. Box 4956, 0424 Oslo, Norway 


\section{Introduction}

More than $70 \%$ of patients with schizophrenia are estimated to experience cognitive impairments [1]. For several cognitive domains-including verbal learning and memory, attention, perception, and processing speed-the impairment may be as prominent as two standard deviations below healthy controls [2] indicating a clinically relevant decrease or deficits in cognitive functioning. These deficits can have a more substantial impact than positive psychotic symptoms in determining real-world functional outcome [3, 4], and may be present even after successful reduction in psychotic symptoms [5, 6]. Although considerable efforts have been made to understand the neurobiology of cognitive impairment in psychosis, the background is still mostly undetermined.

Lipids are essential for CNS development and functioning [7-10], including myelination and synapse communication. In schizophrenia and related psychotic disorders, both lipid disturbances as well as brain structural changes have been demonstrated. While several studies in the normal aging population, as well as in other neuropsychiatric disorders [11-17], characterized by compromised brain functioning have indicated a link between serum cholesterol levels, in particular HDL cholesterol, and cognition, few studies have examined the potential relationship between serum lipids and cognition in patients with schizophrenia. In a cross-sectional study, Lancon et al. [18] found low HDL levels (along with hypertriglyceridemia, and abdominal obesity) to be related to impairment in verbal learning and memory. Another cross-sectional study [19] demonstrated that patients with schizophrenia and co-morbid metabolic syndrome exhibited impairments in processing speed, attention/vigilance, working memory and problem solving, compared to those without metabolic syndrome. However, when the authors examined the different components of the metabolic syndrome, they found that the HDL level was positively associated with attention/vigilance. Also, studies in antipsychotic-treated patients have reported a link between serum lipids and cognitive performance. Krakowski and colleagues found that an increase in total cholesterol level and LDL level after a 12-week period of antipsychotic drug treatment was positively associated with improvements in global cognition [20] with verbal memory being the most prominent domain. Still, there are other studies in schizophrenia that have failed to show any associations between serum lipids and cognition [21, 22], and some have even found negative associations between serum lipids and cognitive performance [23].

These discrepancies in prior studies may be due to the potential confounding effects of long-term medication, age-related neurodegeneration and disease risk-related environmental factors, as most previous studies have been focusing on chronic schizophrenia patients in a cross-sectional study design. Another important limitation is a lack of age- and gender-matched healthy individuals to control for practice effects [24] and naturally occurring variations in serum lipid levels.

\section{Aims of the study}

The present longitudinal study of an FEP cohort was undertaken to investigate potential associations between serum lipid levels and cognitive performance in the first year of antipsychotic treatment in a group of FEP patients with limited prior drug exposure, compared to a group of age- and gender-matched healthy controls (HC). We further investigated the possible influence of lifestyle (smoking, alcohol, diet, and exercise), metabolic measures connected to serum lipids (body mass index), improvements in clinical symptomatology secondary to antipsychotic drug treatment, and other illness-related factors (antipsychotic drug treatment, hospitalization, and depressive symptoms) on the relationship between serum lipids and cognition in the patient group.

\section{Methods and materials}

\section{Participants}

The longitudinal FEP sample, recruited between 2003 and 2013 through the Thematically Organized Psychosis (TOP) study (Oslo, Norway), was the same as used by Gjerde et al. [25]. The inclusion criteria for the TOP study were age between 18 and 65 years, and having a diagnosis within a broad schizophrenia spectrum psychosis according to the Diagnostic and Structural Manual of Mental Disorders, fourth version (DSM-IV, American Psychiatric Association, 2000). Participants were recruited in a stable phase of their disorder. Exclusion criteria were current IQ under 70 , the presence of a neurological disorders or a history of head injuries. Additionally, for the longitudinal FEP study, patients who previously had received adequate antipsychotic drug treatment were not recruited [25]. Healthy controls (HC) were randomly selected and recruited from the same geographic catchment area as the patients, using national statistical records (2003-2011). They were matched for age and gender. To ensure a reliable $\mathrm{HC}$ sample, subjects were excluded if they or any of their first-degree relatives had a history of a severe psychiatric disorder as indicated by the Primary Care Evaluation of Mental Disorders [26]. The final sample consisted of 132 first episode psychosis (FEP) patients and $83 \mathrm{HC}$. Participants were tested at baseline 
and after 1 year. None of the participants used medication against thyroid problems, diabetes, hypertension or cholesterol-lowering medications (statins or fibrates).

All participants gave written informed consent prior to their inclusion in the study, and the Regional Ethics Committee and The Norwegian Data Inspectorate approved the study.

\section{Clinical assessments}

Demographic and clinical data (including antipsychotic drug treatment) were obtained through structured interviews and from hospital medical records. The diagnosis was established by use of the Structural Clinical Interview for DSMIV (SCID) [27]. Psychotic symptoms were assessed with the Positive and Negative Syndrome Scale (PANSS) [28] and depressive symptoms with the Calgary Depression Scale for Schizophrenia (CDSS) [29]. Remission was defined as at least 1 week with no score of $\geq 4$ on any of the following five PANSS items: P1, P3, P5, P6, or G9. Alcohol use was measured with the Alcohol Use Disorders Identification Test (AUDIT) [30]. Information on diet and exercise habits for the FEP patients was obtained by self-assessment at baseline and follow-up. Patients were asked to describe their eating habits as healthy, moderately healthy or unhealthy, while exercise was initially assessed as light, moderate or hard. Diet was then dichotomized into "similar or poorer diet" (including patients who did not change their diet habits from inclusion to 12 months, as well as patients who reported a poorer diet at 12 months) and "better diet" (consisting of patients who reported a beneficial change in diet). Exercise was likewise dichotomized into "similar or reduced exercise" and "increased exercise" at 12 months (for more details, see [25]).

\section{Antipsychotic drug treatment in the FEP group}

As we did not have sufficient statistical power to create separate subgroups for the different antipsychotic agents (see supplementary Tables 1, 2 and 3 for antipsychotic use), we

Table 1 Demographic, metabolic, and cognitive characteristics of the FEP and HC sample

\begin{tabular}{|c|c|c|c|c|c|c|}
\hline & $\operatorname{FEP}(n)$ & $\mathrm{HC}(n)$ & FEP, mean (SD) & $\mathrm{HC}$, mean $(\mathrm{SD})$ & $t$ & $p$ value \\
\hline Age (years) & 132 & 83 & $26.7(7.6)$ & $29.0(6.8)$ & -2.6 & 0.01 \\
\hline Education (years) & 132 & 83 & $12.9(2.8)$ & $14.2(2.0)$ & -4.43 & $<0.001$ \\
\hline Alcohol use (AUDIT scores) & 120 & 71 & $7.3(7.4)$ & $5.9(3.0)$ & 1.89 & 0.06 \\
\hline DUP (weeks), median (range) & 132 & & $40.0(1-1040)$ & & & \\
\hline Total PANSS scores & 132 & & $63.2(13.9)$ & & & \\
\hline PANSS-positive subscores & 132 & & $2.6(1.0)$ & & & \\
\hline PANSS-negative subscores & 132 & & $2.2(1.0)$ & & & \\
\hline \multirow[t]{2}{*}{ Depressive symptoms (CDSS scores) } & 128 & & $6.2(4.5)$ & & & \\
\hline & $\operatorname{FEP}(n)$ & $\mathrm{HC}(n)$ & $\mathrm{FEP}, n(\%)$ & $\mathrm{HC}, n(\%)$ & $X^{2}$ & $p$ value \\
\hline Gender (male) & 132 & 83 & $85(64.4)$ & $80(59.7)$ & 0.62 & 0.45 \\
\hline Ethnicity (Caucasian) & 132 & 83 & $87(65.9)$ & $132(98.3)$ & 52.32 & $<0.001$ \\
\hline Smoking (daily) & 132 & 86 & $60(45.5)$ & $17(19.8)$ & 15.04 & $<0.001$ \\
\hline Hospitalization at inclusion & 132 & & $30(22.7)$ & & & \\
\hline Change in diet ("better diet") ${ }^{\mathrm{a}}$ & 132 & & $20(15.2)$ & & & \\
\hline Change in exercise ("increased exercise ") & 129 & & $21(15.9)$ & & & \\
\hline Continuous antipsychotic drug use ${ }^{c}$ & 132 & & $100(76)$ & & & \\
\hline Intermittent antipsychotic drug use ${ }^{\mathrm{d}}$ & 132 & & $32(24)$ & & & \\
\hline Antipsychotic monotherapy & 132 & & $91(69)$ & & & \\
\hline Antipsychotic polypharmacy & 132 & & $41(31)$ & & & \\
\hline
\end{tabular}

$p$ values are obtained from $T$ tests and chi-square test

$p$ values in bold indicate numbers that are significant on the $95 \%$ confidence limit

FEP first episode psychosis, $H C$ healthy controls, $S D$ standard deviation, $n$ number of subjects, \% percentage, AUDIT Alcohol Use Disorder Identification Test, DUP duration of untreated psychosis, PANSS Positive and Negative Syndrome Scale, CDSS Calgary Depression Scale for Schizophrenia

aDiet was divided into "similar or poorer" and "better" compared to diet habits at baseline

bexercise was divided into "similar or reduced" and "increased" compared to exercise habits at baseline

"Continuous antipsychotic drug use" refers to the continuous use of any antipsychotic agent during the study period

d“Intermittent antipsychotic drug use" refers to the use of any antipsychotic agent, either at baseline or 12 months 
Table 2 Mixed models examining serum lipids, BMI and cognitive performance at baseline in FEP and $\mathrm{HC}$
Table 3 Mixed models examining serum lipid, BMI and cognitive trajectories over 1 year in FEP and HC

\begin{tabular}{|c|c|c|c|c|c|c|}
\hline & \multirow[t]{2}{*}{ FEP, mean (SD) } & \multirow[t]{2}{*}{$\mathrm{HC}$, mean $(\mathrm{SD})$} & \multicolumn{2}{|l|}{ Group } & \multicolumn{2}{|l|}{ Group } \\
\hline & & & $F$ & $p$ value & $B$ & $\mathrm{SE}(B)$ \\
\hline Total cholesterol (mmol/L) & $4.97(1.00)$ & $4.59(0.90)$ & 8.94 & 0.003 & 0.39 & 0.13 \\
\hline $\mathrm{HDL}(\mathrm{mmol} / \mathrm{L})$ & $1.39(0.40)$ & $1.44(0.43)$ & 0.08 & 0.79 & & \\
\hline LDL (mmol/L) & $2.99(0.82)$ & $2.59(0.81)$ & 11.54 & 0.001 & 0.38 & 0.11 \\
\hline Ln TG (mmol/L) & $0.81(0.31)$ & $0.77(0.26)$ & 0.82 & 0.37 & & \\
\hline BMI $\left(\mathrm{kg} / \mathrm{m}^{2}\right)$ & $24.9(4.3)$ & $24.1(3.7)$ & 1.57 & 0.21 & & \\
\hline Verbal learning (total scores) & $49.31(11.80)$ & $59.22(8.54)$ & 35.25 & $<0.001$ & -8.82 & 1.48 \\
\hline Processing speed (total scores) & $60.40(16.82)$ & $79.55(13.32)$ & 65.19 & $<0.001$ & -16.77 & 2.08 \\
\hline Working memory (total scores) & $9.21(2.61)$ & $11.89(2.40)$ & 48.33 & $<0.001$ & -2.50 & 0.36 \\
\hline Verbal fluency (total scores) & $35.58(14.54)$ & $45.39(9.98)$ & 18.27 & $<0.001$ & -7.65 & 1.79 \\
\hline Inhibition (total scores) & $66.07(24.14)$ & $49.52(10.83)$ & 27.75 & $<0.001$ & 15.08 & 2.86 \\
\hline
\end{tabular}

$p$ values in bold indicate numbers that are significant on the $95 \%$ confidence limit

Verbal learning was measured with California Verbal Learning Test, processing speed with Digit Symbol Test, working memory with Letter number span, verbal fluency with Verbal Fluency Test, and inhibition with Color-Word Interference Test. Analyzed with mixed-effects models while controlling for age, gender, education, and group (FEP vs. HC). The HC were defined as the reference group

$F E P$ first episode psychosis, $H C$ healthy controls, $S D$ standard deviation, $F F$ value, $B$ regression coefficient, $S E$ standard error of the regression coefficient, $H D L$ high-density lipoprotein cholesterol, $L D L$ lowdensity lipoprotein cholesterol, $L n T G \mathrm{Ln}$ of triglycerides, $B M I$ body mass index

\begin{tabular}{|c|c|c|c|c|c|c|}
\hline & \multicolumn{2}{|l|}{ Time } & \multicolumn{2}{|c|}{ Group $\times$ time } & \multicolumn{2}{|c|}{ Group $\times$ time } \\
\hline & $F$ & $p$ value & $F$ & $p$ value & $B$ & $\mathrm{SE}(B)$ \\
\hline Total cholesterol (mmol/L) & 0.005 & 0.94 & 0.003 & 0.96 & & \\
\hline $\mathrm{HDL}(\mathrm{mmol} / \mathrm{L})$ & 2.77 & 0.10 & 2.24 & 0.14 & & \\
\hline $\mathrm{LDL}(\mathrm{mmol} / \mathrm{L})$ & 0.32 & 0.57 & 1.48 & 0.23 & & \\
\hline $\mathrm{Ln} \mathrm{TG}(\mathrm{mmol} / \mathrm{L})$ & 0.05 & 8.39 & 0.05 & 0.82 & & \\
\hline $\operatorname{BMI}\left(\mathrm{kg} / \mathrm{m}^{2}\right)$ & 23.84 & $<0.001$ & 6.99 & 0.01 & 0.95 & 0.36 \\
\hline Verbal learning (total scores) & 17.60 & $<0.001$ & 6.86 & 0.01 & 3.54 & 1.35 \\
\hline Processing speed (total scores) & 0.05 & 0.003 & 2.27 & 0.13 & & \\
\hline Working memory (total scores) & 0.10 & 0.75 & 0.10 & 0.75 & & \\
\hline Verbal fluency (total scores) & 0.21 & 0.65 & 6.38 & 0.01 & -3.15 & 1.25 \\
\hline Inhibition (total scores) & 0.60 & 0.02 & 0.37 & 0.54 & & \\
\hline
\end{tabular}

$p$ values in bold indicate numbers that are significant on the $95 \%$ confidence limit

Verbal learning was measured with California Verbal Learning Test, processing speed with Digit Symbol Test, working memory with Letter number span, verbal fluency with Verbal Fluency Test, and inhibition with Color-Word Interference Test. Analyzed with mixed-effects models examining a time $\times$ group interaction while controlling for age, gender, education, time, and group (FEP vs. HC). The HC were defined as the reference group

$F E P$ first episode psychosis, $H C$ healthy controls, $F f$ value, $B$ regression coefficient, $S E$ standard error of the regression coefficient, $H D L$ high-density lipoprotein cholesterol, $L D L$ low-density lipoprotein cholesterol, $L n T G \ln$ of triglycerides, $B M I$ body mass index organized data on antipsychotic medication as "continuous antipsychotic drug use" versus "intermittent antipsychotic drug use" [25]. Continuous use was defined as using any antipsychotic agent at baseline and at 12 months (not necessarily the same agent at both time points), while intermittent use was defined as using any antipsychotic agent at either baseline or at 12 months. In addition, we differentiated between antipsychotic monotherapy (using only one antipsychotic at the specific time point) and polypharmacy (using two or more antipsychotic agents at the same time point). All patients who used first-generation drugs also used second-generation drugs, and due to insufficient information regarding switches and duration of antipsychotic treatments during the follow-up period, it was not possible to calculate cumulative drug exposure. 


\section{Cognitive tests}

The cognitive test battery is comprised of five cognitive domains (verbal learning, processing speed, working memory, verbal fluency, and inhibition) based on previous findings related to cognitive dysfunction in FEP patients [31].

Psychologists trained in standardized neuropsychological testing carried out the cognitive assessment. Current IQ was estimated from the Wechsler Adult Intelligence Scale (WASI); subscale similarities and block design. Verbal learning was measured with the California Verbal Learning Test (CVLT-II) [32], verbal learning sub-score. Processing speed was measured with the Digit Symbol Test [33]. Working memory was measured with the Letter number sequencing test [33]. Verbal fluency was measured with the Verbal Fluency Test, phonetic sub-score from the Delis-Kaplan Executive Functioning System (D-KEFS) [34]. Finally, inhibition was measured with the Color-Word Interference Test from the D-KEFS [34], inhibition sub-score. Raw scores were reported for all tests to be able to calibrate the cognitive performance of the FEP patients compared to a representative age- and gender-matched $\mathrm{HC}$ group from the same catchment area as the patients. Higher scores indicated better performance on all tests except for the inhibition sub-test where higher scores indicated poorer performance. All participants showed an adequate neuropsychological test effort indicated by two errors or less on the forced recognition trial of the California Verbal Learning Task (CVLT-II) [32].

\section{Serum lipids and BMI}

Blood was drawn from the antecubital vein in the morning after overnight fasting at baseline and 12 months, on the same day as the neuropsychological testing took place (time period: 2003-2013). The Department of Clinical Chemistry at Oslo University Hospital, using standard enzymatic methods from Roche Diagnostics Norge AS (Oslo, Norway), analyzed cholesterol (total, HDL, LDL) and triglycerides (TG). All blood samples were sent immediately after collection and analyzed continuously as routine samples. Digital weights were used to weight all the participants and body mass index (BMI) calculated accordingly: $\mathrm{BMI}=$ weight (in kilograms) divided by height (in meters squared) $\left(\mathrm{kg} / \mathrm{m}^{2}\right)$.

\section{Statistical analyses}

The data were analyzed with SPSS version 23 (SPSS Inc., Chicago, IL, USA/IBM, New York, USA). The level of statistical significance was preset to $p<0.05$ (two tailed). For the comparison of demographic characteristics, chi-square tests and $t$ tests were used as appropriate.

For the main analyses, we employed linear mixed models with unstructured covariance matrix to examine longitudinal changes and between-group effects of lipid measures on cognitive measures. Preliminary analyses were conducted to ensure assumptions of normality, linearity, multicollinearity, and homoscedasticity. Serum TG values were lntransformed after inspection of residuals. Due to issues with multicollinearity, we did not enter all the lipids in the same mixed model analysis. There were no problems with linearity or homoscedasticity.

In separate mixed models, we first examined any baseline differences in metabolic and cognitive measures controlling for age, gender, and education. Then, we explored the 1-year changes in serum lipids, BMI, and cognitive performance. In the latter longitudinal models, we also included time and a group (FEP and HC) $\times$ time interaction.

Our approach to the interpretation of statistical results obtained from the cognitive models was that a main effect of time would indicate cognitive changes due to drug treatment or other causes (i.e., practice effects). To disambiguate the latter two possibilities, we would compare the cognitive performance of the patient group with that of the HC group. Group $\times$ time interactions that favored steeper improvement in the FEP group would be viewed as an indication of a possible treatment effect, reflecting cognitive enhancement. A main effect for time in the absence of such an interaction could be regarded as representing practice effects.

The mixed models that displayed a significant group $\times$ time interaction were subsequently examined for a potential relationship with serum lipids. The rationale for this was that group-specific changes to cognition might be related to alterations in serum lipids due to antipsychotic drug treatment. In these mixed models, the scores from the specific cognitive tests were defined as dependent variables, while time (baseline and 12 months), age, gender, years of education, group affiliation (FEP or HC), and serum lipids were entered as independent variables. To investigate if there was a group-dependent effect of lipids on cognition, we also included a group $\times$ lipid interaction.

In post hoc analyses, using similar mixed models as in our main analyses, we further examined if any groupspecific lipid effects on cognition could be explained by ethnicity, lifestyle-related factors (smoking, alcohol, diet, and exercise), concomitant changes in BMI, be secondary to antipsychotic treatment effects on positive and negative symptoms, remission status at 12 months, or be explained by other illness-related factors (hospitalization and depressive symptoms).

\section{Missing data}

Preliminary analyses indicated a random distribution of the missing variables and that there were no significant differences in sociodemographic characteristics or cognitive test scores between the subjects with a complete dataset on 
serum lipids compared to the ones with missing data (Little's MCAR test: chi-square $=1912.51, d f=1865, p=0.22$ ). Forty-seven (36\%) of the FEP patients lacked cognitive data at follow-up. These FEP patients were included in the main analyses because they had baseline data and a mixed linear model approach using all available information was employed. As a precaution, we also checked the validity of our results by repeating the analyses excluding participants with an incomplete dataset.

\section{Results}

For a detailed description of demographic characteristics and group comparison of FEP with HC, see Table 1. In summary, the FEP patients had significantly shorter education, more non-Caucasian ethnicity and a higher percentage of daily smokers, compared to the HC.

\section{Group differences in serum lipids at baseline and after 1 year of follow-up}

As described in Table 2, the FEP patients had significantly higher levels of total cholesterol and LDL compared to the $\mathrm{HC}$ at baseline, $(F=8.94, p=0.003)$ and $(F=11.54$, $p=0.001)$, respectively. During the follow-up, there were no significant changes in mean levels of serum lipids among the FEP patients when compared to the $\mathrm{HC}$ at the group level (Table 3). Still, there were individual differences, and at the individual level the serum lipid changes for the FEP were the following: 47 participants (47\%) experienced a decrease and 52 participants (53\%) an increase in their total cholesterol levels; 49 participants (54\%) experienced a decrease and 41 participants (46\%) an increase in their HDL levels; 43 participants (50\%) experienced a decrease and 43 participants (50\%) an increase in their LDL levels; 40 participants (45\%) experienced a decrease and 49 participants (55\%) an increase in their TG levels.

\section{Group differences in cognition at baseline and after 1 year of follow-up}

The FEP patients demonstrated marked impairments in cognitive functioning across all domains at baseline $(p<0.001)$ (Table 2). During the 1-year follow-up, there was a main effect of time $(F=17.60, p<0.001)$ (indicating significant changes in cognitive performance from baseline to 12 months) as well as a group by time interaction for verbal learning $(F=6.86, p=0.01)$, with a steeper change in FEP compared to $\mathrm{HC}(B=3.54, p=0.01)$, indicating that practice effects alone cannot explain the relatively higher improvement in patients compared to HC. The mixed model analyses also showed a group by time interaction for verbal fluency ( $F=6.38, p=0.01)$; here, the FEP group exhibited a deterioration in comparison with the HC group, $(B=-3.15$, $p=0.01$ ), respectively.

\section{Association between serum lipids and cognition}

When examining the two cognitive domains that showed a group by time interaction (verbal learning and verbal fluency) for associations with serum lipid levels, we found that there was a significant group by HDL interaction effect for verbal learning $(F=11.12, p=0.001)$, where an increase in HDL level was related to improvement in verbal learning among FEP patients compared to the $\mathrm{HC}(B=10.32$, $p=0.001$ ). Verbal fluency, in contrast, did not evidence any significant group by lipid interactions. Follow-up analyses excluding patients with incomplete cognitive datasets produced comparable results (data not shown).

Because the FEP patients, in contrast to HC, included several subjects with their primary education outside Norway, which could influence the cognitive test scores, we also reran the analyses without these patients and obtained similar results as in our main analyses (data not shown).

\section{Post hoc analyses exploring the significant associations found between serum lipids and cognition}

The post hoc mixed models controlling for antipsychotic usage (i.e., continuous use vs. intermittent use), and positive and negative symptoms indicated that the group (i.e., FEP)specific link between HDL and verbal learning remained highly significant $(F=7.45, p=0.007)$. A significant main effect of positive symptoms $(F=4.67, p=0.03)$ and negative symptoms $(F=11.77, p=0.001)$, but not for antipsychotic drug use $(F=1.03, p=0.31)$, was also exhibited. As the magnitude of the effect is reflected in the standardized regression coefficients, we examined these coefficients to discern whether HDL cholesterol had a stronger impact than negative and positive symptoms on verbal learning. Our results indicated that HDL $(B=3.13, p=0.007)$ was a stronger predictor for verbal learning than were negative $(B=-2.20, p=0.001)$ and positive symptoms $(B=-1.76$, $p=0.03$ ). Likewise, controlling for remission status did not significantly impact the observed link between serum HDL and verbal learning in the FEP group $(B=8.42, p=0.002)$.

We further investigated the influence of antipsychotic drug treatment (continuous use vs. intermittent use, and antipsychotic monotherapy vs. polypharmacy) on the relationship between HDL levels and verbal learning. The group (FEP)-specific HDL-link remained highly significant, $(F=10.76, p=0.001)$ and $(F=10.54, p=0.001)$, whereas the main effect of antipsychotic drug treatment was absent, 
inferring limited value to differential treatment effects on the observed link between HDL and verbal learning.

Furthermore, though there was a main effect of BMI on verbal learning $(F=4.88, p=0.03)$, the group by HDL interaction remained significant $(F=9.86, p=0.002)$ indicating that the link between HDL and verbal learning was independent of BMI. Of note, ethnicity, hospitalization, depressive symptoms, smoking habits, diet, exercise, and alcohol use demonstrated no attenuation of results for the observed link between HDL and verbal learning (data not shown).

\section{Discussion}

The current study of antipsychotic-treated FEP patients showed that during the first year of follow-up, an increase in serum HDL was associated with better verbal learning capacity. This relationship was independent of practice effect (as controlled by a group of age- and gender-matched HC), lifestyle, concurrent changes in BMI, and improvements in clinical symptomatology. Having in mind that cognitive performance is an important predictor of longitudinal clinical and functional outcomes in patients with psychotic disorders, our findings may be of clinical relevance.

Consistent with prior literature [35], the FEP patients performed poorer on all cognitive tests compared to the HC. During the follow-up, there was a group-specific improvement in verbal learning, which subsequent analyses showed was associated with an increase in HDL levels among the FEP patients but not amongst HC. Our findings are in line with Lancon and colleagues [18] finding a positive association between HDL and verbal learning and memory in a cross-sectional sample of chronic schizophrenia patients. The present study is, however, the first to report such an association in a cohort of FEP patients with age- and gendermatched healthy controls.

The observed link between HDL and cognition is also consistent with the mechanisms that relate lipids to the brain while also agreeing with evidence of impaired lipid metabolism (including cholesterol) in psychotic patients [36-40]. Lipids are not only essential for myelination; synaptic growth and regeneration also depend greatly on the availability of brain cholesterol [41, 42]. Depletion of cholesterol in tissue cultures has, for example, been shown to inhibit synaptogenesis and causes neurodegenerative changes [42]. Brain structures such as the hippocampus express high transcript levels of lipid-biosynthesizing enzymes [42], are densely populated by synapses, and play a prominent role in verbal learning and memory [43, 44]. Several studies have found a positive link between performances on verbal learning and hippocampal volumes [45-47], as well as on distinct subfield volumes [48]. More importantly, a growing number of evidence in the normal aging population and patients with neuropsychiatric disorders, points to high serum HDL levels, in particular, being protective against hippocampal atrophy [49]. This dependency of the hippocampus on intact cholesterol metabolism may at least in parts explain our finding of a distinct link between HDL cholesterol and the hippocampus-based task of verbal learning. While studies are reporting a correlation between different cognitive domains within an individual [50-55], there are several investigators that have emphasized a cognitive architecture best described by relatively independent cognitive domains [2, 56-58], and verbal learning is recognized as one such independent cognitive dimension. In further support of distinguishable cognitive dimensions is the fact that pharmacological interventions, including antipsychotic drug treatment, have been shown to cause changes in specific cognitive domains [59], indicating a pharmacological dissociation of certain groups of cognitive processes.

For decades, the traditional view has been that the cholesterol in the brain is synthesized de novo; newer evidence, however, suggests that excessive cholesterol synthesis may be regulated by intricate efflux mechanisms linking central and peripheral cholesterol levels [60-63]. Indeed, serum HDL has been found to be highly correlated with HDL in cerebrospinal fluid [64]. Still, the nature of this relationship, between serum lipids and lipids in the CNS, is not fully elucidated. It is, however, likely that long-term lipid status may have a different impact on brain structure and functioning than the short-term impacts observed in treatment studies $[19,20,65]$, and that these changes in brain structure and functioning may be more pronounced in the developmental lifespan [66]. While we included a relatively young FEP population (with a mean age of 25 years), future studies may consider including high-risk patients, as well as having a longer study period with frequent observation points.

We recently demonstrated that an increase in HDL during antipsychotic drug treatment was related to an improvement in negative symptoms in the same FEP cohort [25]. Other studies have shown a relationship between negative symptoms and verbal learning [67]. As we now find HDL levels being associated with specific cognitive domains, it is plausible that improvements in cognitive functioning over time were more related to clinical symptom improvement, reflecting "pseudospecificity" [68]. Additionally, lack of motivation and motor retardation inherent in the negative syndrome could also have disturbed test performance in patients with prominent negative symptoms. We attempted to disentangle these phenomena by controlling for secondary changes in negative (and positive) symptoms during antipsychotic drug treatment, obtaining similar results as in our main analyses. Intriguingly, the standardized regression coefficients revealed that the HDL level was more profoundly related to working memory than positive and negative symptoms were, adding support to the growing literature on the role of lipids 
in psychotic disorders. Also, other factors that are known to influence cholesterol metabolism as well as cognitive function such as lifestyle and behavioral factors [69-73] were controlled for in the post hoc analyses, achieving similar results as in our main analyses.

The present study has some additional limitations that need to be addressed. The constitution of the HC group was different from the FEP group since the latter included subjects with their primary education from outside Norway, which could influence cognitive performance especially for verbal tests. We tried to minimize the effect of this limitation by controlling for education in the mixed model analyses. Also, we reran the analyses excluding the patients without a Norwegian education, generating similar results. Information on diet and exercise habits was only available for FEP but not for HC. Additionally, self-assessment about diet and exercise may be problematic as it may be linked to high social desirability; on the other hand, achieving truly reliable data on these parameters may be difficult outside the confinement of an institution. Due to power issues, we were not able to examine specifically and compare different antipsychotic agents and how this may influence our results. Still, our main objective was to examine the effect of serum lipids per se on cognition in FEP patients, and for this particular aim, differentiating the antipsychotic agents might be less prudent. We further recognize that other factors then practice can explain a main effect of time: the controls are healthy, and could in theory be susceptible to a ceiling effect in the amount of change that can be achieved; leading to potential differences in the learning potential in the two groups. While an untreated control group may have been ideal to address this matter, it is not ethically feasible. Finally, as our study is naturalistic in design, temporality cannot be established, and studies with a randomized controlled design, which include treatment targeted explicitly at elevating HDL levels and measurements of change in cognitive performance, are necessary.

In conclusion, the present study of antipsychotic-treated FEP patients showed that during the first year of follow-up, an increase in serum HDL was associated with better cognitive performance. Further research, implementing clinical data with for instance myelin-focused brain imaging techniques, is warranted. As cognitive deficits dramatically impact social and occupational functioning, our findings of a link between HDL and cognition could potentially contribute to the development of new treatment strategies, with functional improvements achieved by increasing HDL levels as an add-on treatment for cognitive remediation that has shown to be effective in patients with psychosis.

Acknowledgements We thank our collaborators in the NORMENT \& KG Jebsen Centre for Psychosis Research, all participants taking part in the study, and Department of Medical Biochemistry, Oslo university hospital for performing the lipid analyses.
Author contributions PBG analyzed the data and was responsible for design of the study, interpretation of results and drafting of the first version of the manuscript together with IM and VMS. OAA, CS, TVL, TU, and NES contributed with data. All authors contributed to the writing of the manuscript and have approved the final version.

Funding The study was supported by grants from the Research Council of Norway to NORMENT CoE (Grant number 223273/F50, under the Centres of Excellence funding scheme) and Stiftelsen Kristian Gerhard Jebsen (SKGJ-MED-008). The funding bodies had no role in the analyses or writing of the manuscript, or the decision to submit this work for publication.

\section{Compliance with ethical standards}

Conflict of interest The authors of this paper have no conflict of interests.

Open Access This article is distributed under the terms of the Creative Commons Attribution 4.0 International License (http://creativeco mmons.org/licenses/by/4.0/), which permits unrestricted use, distribution, and reproduction in any medium, provided you give appropriate credit to the original author(s) and the source, provide a link to the Creative Commons license, and indicate if changes were made.

\section{References}

1. Reichenberg A, Harvey PD, Bowie CR et al (2009) Neuropsychological function and dysfunction in schizophrenia and psychotic affective disorders. Schizophr Bull 35:1022-1029. https://doi. org/10.1093/schbul/sbn044

2. Nuechterlein KH, Barch DM, Gold JM et al (2004) Identification of separable cognitive factors in schizophrenia. Schizophr Res 72:29-39. https://doi.org/10.1016/j.schres.2004.09.007

3. Green MF, Kern RS, Braff DL, Mintz J (2000) Neurocognitive deficits and functional outcome in schizophrenia: are we measuring the "right stuff"? Schizophr Bull 26:119-136

4. Shamsi S, Lau A, Lencz T et al (2011) Cognitive and symptomatic predictors of functional disability in schizophrenia. Schizophr Res 126:257-264. https://doi.org/10.1016/j.schres.2010.08.007

5. McGurk SR, Mueser KT, Walling D et al (2004) Cognitive functioning predicts outpatient service utilization in schizophrenia. Ment Health Serv Res 6:185-188

6. Nuechterlein KH, Subotnik KL, Ventura J et al (2012) The puzzle of schizophrenia: tracking the core role of cognitive deficits. Dev Psychopathol 24:529-536. https://doi.org/10.1017/S095457941 2000132

7. Davletov B, Montecucco C (2010) Lipid function at synapses. Curr Opin Neurobiol 20:543-549. https://doi.org/10.1016/j. conb.2010.06.008

8. Dietschy JM, Turley SD (2004) Thematic review series: brain Lipids. Cholesterol metabolism in the central nervous system during early development and in the mature animal. J Lipid Res 45:1375-1397. https://doi.org/10.1194/jlr.R400004-JLR200

9. Horacek J, Bubenikova-Valesova V, Kopecek M et al (2006) Mechanism of action of atypical antipsychotic drugs and the neurobiology of schizophrenia. CNS Drugs 20:389-409

10. Schmitt S, Castelvetri LC, Simons M (2015) Metabolism and functions of lipids in myelin. Biochim Biophys Acta 1851:999 1005. https://doi.org/10.1016/j.bbalip.2014.12.016

11. Crichton GE, Elias MF, Davey A et al (2014) Higher HDL cholesterol is associated with better cognitive function: the 
Maine-Syracuse study. J Int Neuropsychol Soc 20:961-970. https ://doi.org/10.1017/S1355617714000885

12. Ihle A, Gouveia ER, Gouveia BR et al (2017) High-density lipoprotein cholesterol level relates to working memory, immediate and delayed cued recall in Brazilian older adults: the role of cognitive reserve. Dement Geriatr Cogn Disord 44:84-91. https://doi. org/10.1159/000477846

13. Raffaitin C, Feart C, Le Goff M et al (2011) Metabolic syndrome and cognitive decline in French elders: the Three-City Study. Neurology 76:518-525. https://doi.org/10.1212/WNL.0b013e3182 $0 \mathrm{~b} 7656$

14. Bonarek M, Barberger-Gateau P, Letenneur L et al (2000) Relationships between cholesterol, apolipoprotein E polymorphism and dementia: a cross-sectional analysis from the PAQUID study. Neuroepidemiology 19:141-148. https://doi.org/10.1159/00002 6249

15. Merched A, Xia Y, Visvikis S et al (2000) Decreased high-density lipoprotein cholesterol and serum apolipoprotein AI concentrations are highly correlated with the severity of Alzheimer's disease. Neurobiol Aging 21:27-30

16. Singh-Manoux A, Gimeno D, Kivimaki M et al (2008) Low HDL cholesterol is a risk factor for deficit and decline in memory in midlife: the Whitehall II study. Arterioscler Thromb Vasc Biol 28:1556-1562. https://doi.org/10.1161/ATVBAHA.108.163998

17. van Exel E, de Craen AJM, Gussekloo J et al (2002) Association between high-density lipoprotein and cognitive impairment in the oldest old. Ann Neurol 51:716-721. https://doi.org/10.1002/ ana. 10220

18. Lancon C, Dassa D, Fernandez J et al (2012) Are cardiovascular risk factors associated with verbal learning and memory impairment in patients with schizophrenia? A cross-sectional study. Cardiovasc Psychiatry Neurol 2012:204043. https://doi. org/10.1155/2012/204043

19. Lindenmayer JP, Khan A, Kaushik S et al (2012) Relationship between metabolic syndrome and cognition in patients with schizophrenia. Schizophr Res 142:171-176. https://doi.org/10.1016/j. schres.2012.09.019

20. Krakowski M, Czobor P (2011) Cholesterol and cognition in schizophrenia: A double-blind study of patients randomized to clozapine, olanzapine and haloperidol. Schizophr Res 130:27-33. https://doi.org/10.1016/j.schres.2011.04.005

21. Goughari AS, Mazhari S, Pourrahimi AM et al (2015) Associations between components of metabolic syndrome and cognition in patients with schizophrenia. J Psychiatr Pract 21:190-197. https ://doi.org/10.1097/PRA.0000000000000065

22. Takayanagi Y, Cascella NG, Sawa A, Eaton WW (2012) Diabetes is associated with lower global cognitive function in schizophrenia. Schizophr Res 142:183-187. https://doi.org/10.1016/j.schre s.2012.08.034

23. Boyer L, Richieri R, Dassa D et al (2013) Association of metabolic syndrome and inflammation with neurocognition in patients with schizophrenia. 210:381-386. https://doi.org/10.1016/j.psych res.2013.06.020

24. Goldberg TE, Goldman RS, Burdick KE et al (2017) Cognitive improvement after treatment with second-generation antipsychotic medications in first-episode schizophrenia. Arch Gen Psychiatry 64:1115-1122

25. Gjerde PB, Dieset I, Simonsen C et al (2017) Increase in serum HDL level is associated with less negative symptoms after one year of antipsychotic treatment in first-episode psychosis. Schizophr Res. https://doi.org/10.1016/j.schres.2017.10.042

26. Spitzer RL, Kroenke K, Williams JB (1999) Validation and utility of a self-report version of PRIME-MD: the PHQ primary care study. Primary Care Evaluation of Mental Disorders. Patient Health Questionnaire. JAMA 282:1737-1744
27. Narr KL, Bilder RM, Toga AW et al (2005) Mapping cortical thickness and gray matter concentration in first episode schizophrenia. Cereb Cortex 15:708-719. https://doi.org/10.1093/cerco r/bhh172

28. Kay SR, Fiszbein A, Opler LA (1987) The positive and negative syndrome scale (PANSS) for schizophrenia. Schizophr Bull $13: 261-276$

29. Addington D, Addington J, Maticka-Tyndale E, Joyce J (1992) Reliability and validity of a depression rating scale for schizophrenics. Schizophr Res 6:201-208

30. Saunders JB, Aasland OG, Babor TF et al (1993) Development of the alcohol use disorders identification test (AUDIT): WHO collaborative project on early detection of persons with harmful alcohol consumption-II. Addiction 88:791-804

31. Simonsen C, Faerden A, Romm KL et al (2017) Early clinical recovery in first-episode psychosis: symptomatic remission and its correlates at 1-year follow-up. Psychiatry Res 254:118-125. https://doi.org/10.1016/j.psychres.2017.04.050

32. Delis DC, Kramer JH, Kaplan E, Ober BA (2004) California verbal learning test: CVLT-II. Sweden Pearson Assessment, Stockholm

33. Wechsler D (2003) Wechsler adult intelligence scale: WAIS-III. Pearson Assessment, Stockholm

34. Delis DC, Kaplan E, Kramer JH (2005) The Delis-Kaplan executive function system: D-KEFS. Pearson Assessment, Stockholm

35. Mesholam-Gately RI, Giuliano AJ, Goff KP et al (2009) Neurocognition in first-episode schizophrenia: a meta-analytic review. Neuropsychology 23:315-336. https://doi.org/10.1037/a0014 708

36. Bartzokis G (2012) Neuroglialpharmacology: myelination as a shared mechanism of action of psychotropic treatments. Neuropharmacology 62:2137-2153. https://doi.org/10.1016/j.neuro pharm.2012.01.015

37. Catts VS, Fung SJ, Long LE et al (2013) Rethinking schizophrenia in the context of normal neurodevelopment. Front Cell Neurosci 7:60. https://doi.org/10.3389/fncel.2013.00060

38. Hakak Y, Walker JR, Li C et al (2001) Genome-wide expression analysis reveals dysregulation of myelination-related genes in chronic schizophrenia. Proc Natl Acad Sci USA 98:4746-4751. https://doi.org/10.1073/pnas.081071198

39. Takahashi N, Sakurai T, Davis KL, Buxbaum JD (2011) Linking oligodendrocyte and myelin dysfunction to neurocircuitry abnormalities in schizophrenia. Prog Neurobiol 93:13-24. https://doi. org/10.1016/j.pneurobio.2010.09.004

40. Tkachev D, Mimmack ML, Ryan MM et al (2003) Oligodendrocyte dysfunction in schizophrenia and bipolar disorder. Lancet 362:798-805. https://doi.org/10.1016/S0140-6736(03)14289-4

41. Mauch DH, Nägler K, Schumacher S et al (2001) CNS synaptogenesis promoted by glia-derived cholesterol. Science 294:1354-1357. https://doi.org/10.1126/science.294.5545.1354

42. Orth M, Bellosta S (2012) Cholesterol: its regulation and role in central nervous system disorders. Cholesterol 2012:292598. https ://doi.org/10.1155/2012/292598

43. Tamminga CA, Stan AD, Wagner AD (2010) The hippocampal formation in schizophrenia. Am J Psychiatry 167:1178-1193. https://doi.org/10.1176/appi.ajp.2010.09081187

44. Antoniades M, Schoeler T, Radua J et al (2018) Verbal learning and hippocampal dysfunction in schizophrenia: a meta-analysis. Neurosci Biobehav Rev 86:166-175. https://doi.org/10.1016/j. neubiorev.2017.12.001

45. Chepenik LG, Wang F, Spencer L et al (2012) Structure-function associations in hippocampus in bipolar disorder. Biol Psychol 90:18-22. https://doi.org/10.1016/j.biopsycho.2012.01.008

46. Tischler L, Brand SR, Stavitsky K et al (2006) The relationship between hippocampal volume and declarative memory in a 
population of combat veterans with and without PTSD. Ann N Y Acad Sci 1071:405-409. https://doi.org/10.1196/annals.1364.031

47. Guo X, Li J, Wang J et al (2014) Hippocampal and orbital inferior frontal gray matter volume abnormalities and cognitive deficit in treatment-naive, first-episode patients with schizophrenia. Schizophr Res 152:339-343. https://doi.org/10.1016/j.schre s.2013.12.015

48. Mathew I, Gardin TM, Tandon N et al (2014) Medial temporal lobe structures and hippocampal subfields in psychotic disorders: findings from the Bipolar-Schizophrenia Network on Intermediate Phenotypes (B-SNIP) study. JAMA psychiatry 71:769-777. https ://doi.org/10.1001/jamapsychiatry.2014.453

49. Wolf H, Hensel A, Arendt T et al (2004) Serum lipids and hippocampal volume: the link to Alzheimer's disease? Ann Neurol 56:745-748. https://doi.org/10.1002/ana.20289

50. August SM, Kiwanuka JN, McMahon RP, Gold JM (2012) The MATRICS consensus cognitive battery (MCCB): clinical and cognitive correlates. Schizophr Res 134:76-82. https://doi. org/10.1016/j.schres.2011.10.015

51. Allen DN, Huegel SG, Seaton BE et al (1998) Confirmatory factor analysis of the WAIS-R in patients with schizophrenia. Schizophr Res 34:87-94

52. Dickinson D, Iannone VN, Gold JM (2002) Factor structure of the Wechsler Adult Intelligence Scale-III in schizophrenia. Assessment 9:171-180. https://doi.org/10.1177/10791102009002008

53. Dickinson D, Ragland JD, Calkins ME et al (2006) A comparison of cognitive structure in schizophrenia patients and healthy controls using confirmatory factor analysis. Schizophr Res 85:20-29. https://doi.org/10.1016/j.schres.2006.03.003

54. Hill SK, Beers SR, Kmiec JA et al (2004) Impairment of verbal memory and learning in antipsychotic-naive patients with firstepisode schizophrenia. Schizophr Res 68:127-136. https://doi. org/10.1016/S0920-9964(03)00125-7

55. Burton CZ, Vella L, Harvey PD et al (2013) Factor structure of the MATRICS Consensus Cognitive Battery (MCCB) in schizophrenia. Schizophr Res 146:244-248. https://doi.org/10.1016/j. schres.2013.02.026

56. Friis S, Sundet K, Rund BR et al (2002) Neurocognitive dimensions characterising patients with first-episode psychosis. Br J Psychiatry Suppl 43:s85-s90

57. Tulsky DS, Price LR (2003) The joint WAIS-III and WMS-III factor structure: development and cross-validation of a six-factor model of cognitive functioning. Psychol Assess 15:149-162

58. Gladsjo JA, McAdams LA, Palmer BW et al (2004) A six-factor model of cognition in schizophrenia and related psychotic disorders: relationships with clinical symptoms and functional capacity. Schizophr Bull 30:739-754

59. MacKenzie NE, Kowalchuk C, Agarwal SM et al (2018) Antipsychotics, metabolic adverse effects, and cognitive function in schizophrenia. Front Psychiatry 9:622. https://doi.org/10.3389/ fpsyt.2018.00622
60. Vitali C, Wellington CL, Calabresi L (2014) HDL and cholesterol handling in the brain. Cardiovasc Res 103:405-413. https://doi. org/10.1093/cvr/cvu148

61. Heverin M, Meaney S, Lutjohann D et al (2005) Crossing the barrier: net flux of 27-hydroxycholesterol into the human brain. J Lipid Res 46:1047-1052. https://doi.org/10.1194/jlr.M5000 24-JLR200

62. Bjorkhem I, Meaney S (2004) Brain cholesterol: long secret life behind a barrier. Arterioscler Thromb Vasc Biol 24:806-815. https://doi.org/10.1161/01.ATV.0000120374.59826.1b

63. Zhang J, Liu Q (2015) Cholesterol metabolism and homeostasis in the brain. Protein Cell 6:254-264. https://doi.org/10.1007/s1323 8-014-0131-3

64. Fagan AM, Younkin LH, Morris JC et al (2000) Differences in the Abeta40/Abeta42 ratio associated with cerebrospinal fluid lipoproteins as a function of apolipoprotein E genotype. Ann Neurol Soc 48:201-210

65. Meyer JM, Nasrallah HA, McEvoy JP et al (2005) The Clinical Antipsychotic Trials Of Intervention Effectiveness (CATIE) Schizophrenia Trial: clinical comparison of subgroups with and without the metabolic syndrome. Schizophr Res 80:9-18. https:// doi.org/10.1016/j.schres.2005.07.015

66. Keefe RSE (2014) The longitudinal course of cognitive impairment in schizophrenia: an examination of data from premorbid through posttreatment phases of illness. J Clin Psychiatry 75(Suppl 2):8-13. https://doi.org/10.4088/JCP.13065su1.02

67. O'Leary DS, Flaum M, Kesler ML et al (2000) Cognitive correlates of the negative, disorganized, and psychotic symptom dimensions of schizophrenia. J Neuropsychiatry Clin Neurosci 12:4-15. https://doi.org/10.1176/jnp.12.1.4

68. Trampush JW, Lencz T, DeRosse P et al (2015) Relationship of cognition to clinical response in first-episode schizophrenia spectrum disorders. Schizophr Bull 41:1237-1247. https://doi. org $/ 10.1093 / \mathrm{schbul} / \mathrm{sbv} 120$

69. Ashen MD, Blumenthal RS (2005) Clinical practice. Low HDL cholesterol levels. N Engl J Med 353:1252-1260. https://doi. org/10.1056/NEJMcp044370

70. Kramer AF, Erickson KI, Colcombe SJ (2006) Exercise, cognition, and the aging brain. J Appl Physiol 101:1237-1242. https:// doi.org/10.1152/japplphysiol.00500.2006

71. Panza F, Solfrizzi V, Colacicco AM et al (2004) Mediterranean diet and cognitive decline. Public Health Nutr 7:959-963

72. Swan GE, Lessov-Schlaggar CN (2007) The effects of tobacco smoke and nicotine on cognition and the brain. Neuropsychol Rev 17:259-273. https://doi.org/10.1007/s11065-007-9035-9

73. Ward MA, Carlsson CM, Trivedi MA et al (2005) The effect of body mass index on global brain volume in middle-aged adults: a cross sectional study. BMC Neurol 5:23. https://doi. org/10.1186/1471-2377-5-23 